\title{
Hydroclimatic reconstructions over Europe and the Mediterranean
}

\section{EURO-AED2k}

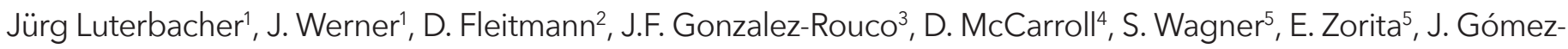 \\ Navarro' ${ }^{6}$ U. Büntgen ${ }^{7,8}$ and J. Esper ${ }^{9}$
}

Meeting of the Euro-Med2k Working Group - Reading, UK, 25-26 March 2013

\begin{abstract}
Water availability has been a crucial constraint on both past societies and ecosystems; therefore identification of historical extreme hydrological events (severity and duration of droughts, magnitude of floods) is important. These characteristics were addressed at a meeting of the PAGES Euro-Med2k Working Group in Reading where a number of presentations highlighted the variability of those extremes, and identified the space- and timescales that are resolved at various European proxy sites.
\end{abstract}

\section{During the first day, participants presented} overviews of high- and low-resolution hydroclimatic proxy records from different archives. Discussion revealed that it is often unclear whether individual proxies reflect moisture availability, soil moisture, precipitation, flood events, drought intervals or some combination. The large variety and different sensitivity of hydrological proxy records thus complicates their integration and the reconstruction of one specific target variable. For instance, a measure of hydroclimate, such as the Palmer Drought Severity Index, is not even well defined for the observational period and different algorithms (e.g. Thornwaite versus Penman-Monteith scheme) may produce markedly different results (van der Schrier et al. 2011).

Further emphasis was also placed on dating issues; distinguishing climatic and non-climatic influences; seasonal biases in proxy records and archive-specific aspects of these proxies that can contribute to reconstruction uncertainties. The potential and limitations of documentary and natural proxies to reconstruct the full range of variations in the regional hydrological cycle were discussed. Discussion centered upon the problems of obtaining a comprehensive overview of the proxy information that is currently available for different aspects of hydroclimate, which is a necessary step before making decisions about how to combine them or translate them into a common target.

It became clear that in contrast to temperature, hydrological parameters have a much shorter de-correlation distance. Thus, without a dense network of precipitation proxies spatially highly resolved reconstructions are limited (Büntgen et al. 2010).

The second day focused on the comparisons between proxy-reconstructed precipitation

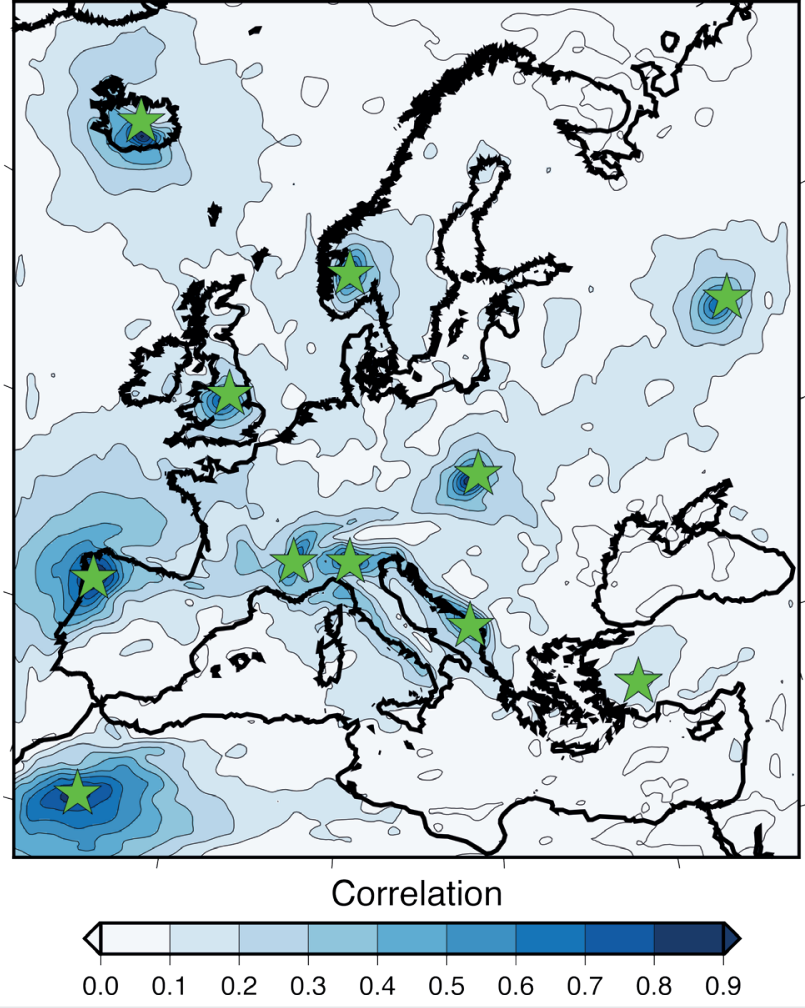

Figure 1: Correlation map between summer precipitation reconstructed using the "analog method" based on the pseudo-proxy locations (green stars) and the original regional climate model output. The reconstructed period is 1-1999 AD (Gomez et al., unpublished data). records and climate model simulations, to assess model veracity and explore mechanisms of internal or externally forced variability contributing to hydroclimate changes. For instance, results from regional climate model simulations for the last two millennia can be used to carry out pseudo-proxy experiments $(P P E)$, i.e. reconstructing the modeled precipitation fields using only the pseudo proxy locations (Fig. 1). A PPE was carried out for precipitation using the "analog method" (Zorita and von Storch 1999) but the short de-correlation distance of precipitation demonstrates that the reconstructions only have skill at the local scale, especially during summer. This is due to the local-scale processes involved in generating summer precipitation. The coupling schemes between soil moisture and atmospheric variables may influence regional hydroclimate independently of the large-scale driving conditions (Gómez-Navarro et al. 2013), so an ensemble of regional models is advisable. Simulations with global models participating in the CMIP5 project indicate that internal unforced modes of climate variability tangle temperature and hydroclimate. A possible, though challenging, strategy could be to reconstruct both temperature and hydroclimate simultaneously.

The next workshop will aim at expanding and improving the $2 k$ proxy database in order to develop more reliable trans-regional synthesis products. Application of modern, more elaborated reconstruction methods that can model the complex spatio-temporal processes in hydroclimatology will be needed to produce gridded products.

\section{AFFILIATIONS}

'Department of Geography, University of Giessen, Germany

2Department of Archaeology, University of Reading, UK ${ }^{3}$ Institute of Geoscience, Complutense University, Madrid, Spain

${ }^{4}$ Department of Geography, Swansea University, UK 5 Institute of Coastal Research, Helmholtz-Zentrum Geesthacht, Germany

${ }^{6} \mathrm{Climate}$ and Environmental Physics and Oeschger Centre for Climate Change Research, University of Bern, Switzerland

'WSL, Birmensdorf, Switzerland

Global Change Research Centre AS CR, Brno, Czech Republic

'Department of Geography, University of Mainz, Germany CONTACT

J. Luterbacher: Juerg.luterbacher@geogr.uni-giessen.de REFERENCES

Büntgen U et al. (2010) Clim Res 41: 125-130

Gómez-Navarro JJ et al. (2013) Clim Past 9: 1667-1682

van der Schrier G et al. (2011) J Geophys Res [Atmos] 116, doi: 10.1029/2010JD015001

Zorita E, von Storch H (1999) J Clim 12: 2474-2489 